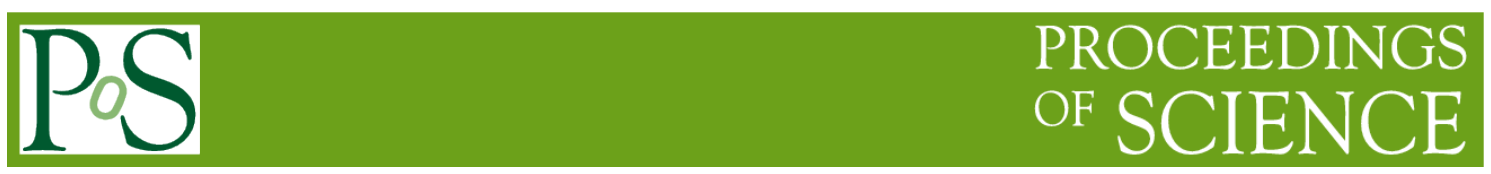

\title{
Highlights from the ANTARES neutrino telescope
}

\author{
Annarita Margiotta ${ }^{1}$ on behalf of the ANTARES Collaboration \\ Dipartimento di Fisica e Astronomia dell'Università e INFN - Bologna \\ v.le C. Berti-Pichat, 6/2, 40127 - Bologna (Italy) \\ E-mail: annarita.margiotta@unibo.it
}

\begin{abstract}
ANTARES is the largest neutrino telescope currently in operation in the Northern hemisphere. Its location and the excellent optical properties of the sea water make it an excellent tool to search for potential emission from the Galactic Centre and the Galactic Plane. The ANTARES physics program includes, among others, the direct search for TeV-PeV neutrinos, indirect searches of dark matter and the detection of signals in coincidence with other cosmic messengers. This document reports a short overview of the most recent and relevant results on this topics, obtained from the analysis of the data recorded by the experiment since 2007 .
\end{abstract}

Neutrino Oscillation Workshop

4 - 11 September, 2016

Otranto (Lecce, Italy)

${ }^{1}$ Speaker 


\section{Introduction}

ANTARES [1] is the largest neutrino telescope in the Northern hemisphere. Its main scientific goal is the detection of astrophysical neutrinos in the TeV to PeV range. ANTARES data have been used to perform searches both for point-like neutrino emitters and for a diffuse flux component, such as the one recently discovered by the IceCube neutrino telescope at the South Pole [2]. Interesting results have been achieved also in the indirect search for dark matter, and in the search for space/time coincidences with other cosmic messengers. Studies on atmospheric neutrinos, magnetic monopoles and other exotic particles, and preliminary studies on the acoustic neutrino detection have also been performed but will not be discussed in this contribution [3].

The ANTARES location (40 km off-shore from Toulon, France), its depth (2475 m under the Mediterranean sea level), and the good optical properties of the seawater, make it an excellent tool for the exploration of the Southern sky, where many gamma-ray sources, possible emitters of astrophysical neutrinos, have been identified. Two basic topologies of events can be registered, depending on neutrino interactions: track-like and shower-like events. The former is produced by charged current interactions of muon neutrinos, characterized by a long muon track that can travel up to few kilometres, crossing the entire active volume of the detector and allows for an excellent angular resolution, better than $0.4^{\circ}$, of the track direction reconstruction. The latter, due to electron neutrino and to all-flavour neutral current interactions, looks like a spherical distribution of light, upward going moving, confined within about ten meters. In this case, the reconstruction strategy can reach an angular resolution of about $3^{\circ}$ and an error on the energy estimate of about $10 \%$.

\section{Search for a diffuse flux of neutrinos}

Searches for a diffuse flux of neutrinos above the atmospheric neutrino background have been performed using events registered by ANTARES between 2007 and 2015, looking for an excess of events at energies larger than about $100 \mathrm{TeV}$. Both track and shower channels have been used. 7 events are observed in the shower channel (2007-2013), when $5 \pm 2$ events are expected from the atmospheric background and $\sim 1.5$ events from the IceCube cosmic signal, depending on the best fit spectral index. In the track-like sample (2007-2015), 19 events are found with an expected background of $13 \pm 4$ events and $\sim 3$ events according to the IceCube flux. The ANTARES sensitivity, obtained combining the two samples, has been evaluated and an upper limit set, see Fig. 1.

\section{Search for point-like sources of neutrinos}

Previous searches for point-like sources considered only track-like events [4]. Recently, a new reconstruction strategy for shower-like events has been developed, achieving angular resolutions below 3 degrees. Track and shower channels are combined in this analysis. The final sample consists of 7269 track-like and 180 shower-like events, for a total livetime of 2424 days between 2007 and 2015. No significant excess was observed, neither in the all-sky search, nor in 
the study of a list of more than 100 candidate sources. Despite its small size, ANTARES has the best world limits in a wide region of the Southern sky, see Fig. 2.

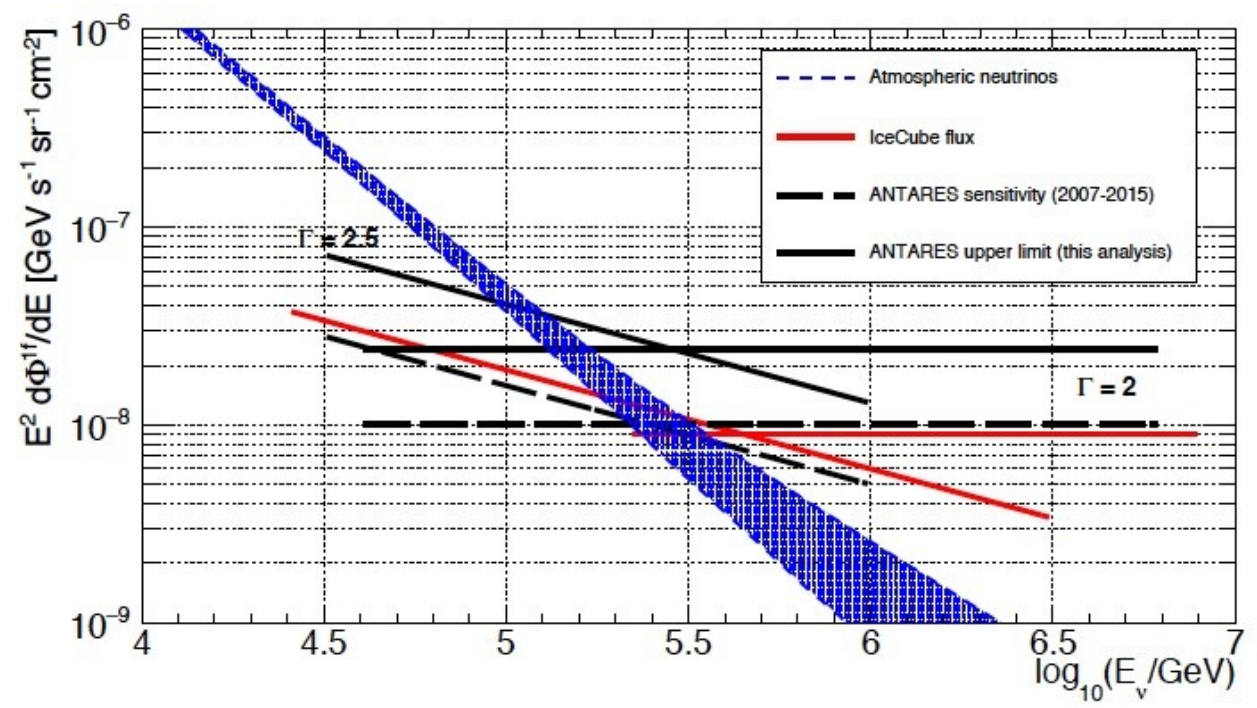

Fig. 1 - ANTARES 90\% C.L. upper limit and sensitivity to a diffuse flux of cosmic neutrinos (track and shower channels combined) for two spectral indexes.

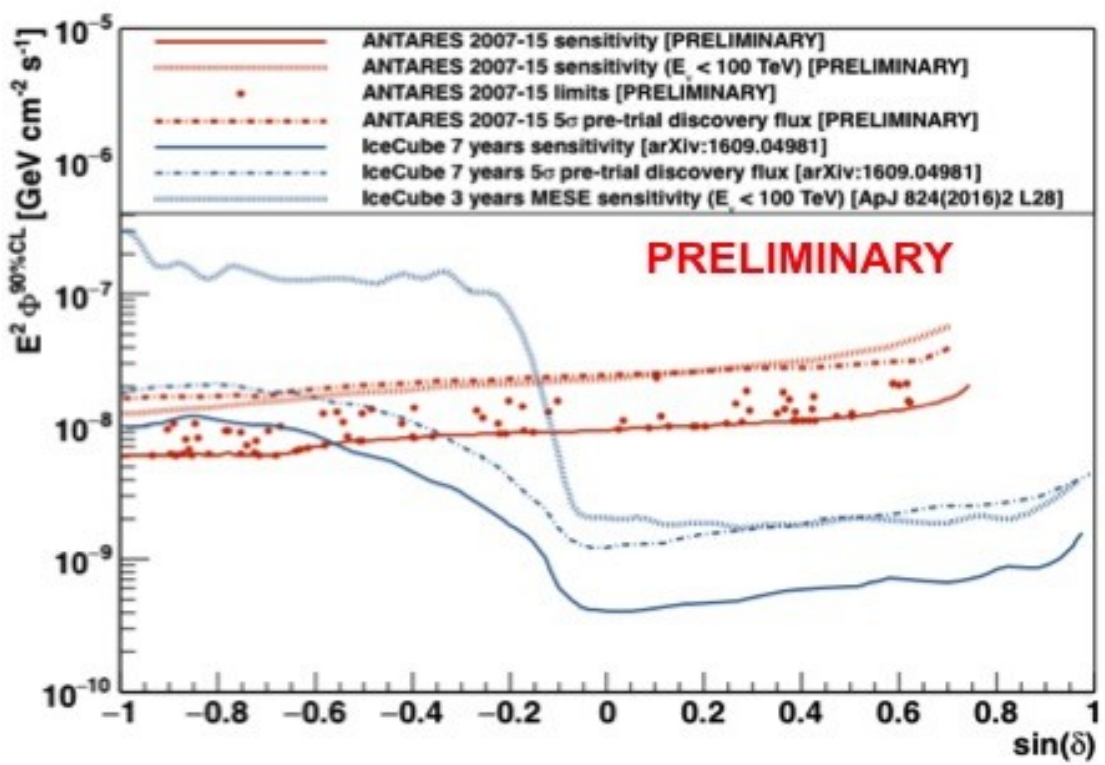

Fig. 2 - ANTARES sensitivity and upper limits ( $90 \%$ C.L.) on the $\mathrm{E}^{-2}$ neutrino flux from selected candidate sources as function of their declination.

\section{Enhanced diffuse flux}

Some regions of the sky are considered possible emitters of neutrinos. A search for neutrino emission from the Fermi Bubbles region (FB) and from a restricted area around the Galactic Plane, the Galactic Ridge, has been performed comparing the number of neutrino events coming from the on-zone and the average number of events from off-zones with equal exposure and extension as the on-zone. This approach does not rely on simulations, reducing the 
effect of systematic uncertainties. No significant excess was found in both cases using track-like events and data from May 2007 to Dec. 2013 for the Galactic ridge, and to Dec. 2015 for the FB [5]. This result puts a constraint on the possible origin of the IceCube sample, excluding that more than 2 IceCube events of the 3yr HESE sample [3] can come from the central area of the Galaxy for spectral indices softer than 2.3. Also, the evaluation of the neutrino flux, simply extrapolating the Fermi gamma-ray flux, has been excluded, see Fig. 3.

\section{Multimessenger approach}

An intense effort has been put in searching for time and/or space coincidences with other cosmic probes. The combination of information provided by the ANTARES neutrino telescope with what is registered by other observatories enhances the probability of detecting a source, allowing the possibility of identifying a neutrino progenitor from a single detected event.

ANTARES has participated in a high-energy (HE) neutrino follow-up of the gravitational wave signal GW150914, providing the first constraint on HE neutrino emission from a binary black hole coalescence [6].

Also, a method based on optical and X-ray follow-ups of high-energy neutrino alerts has been developed within the ANTARES collaboration. The program triggers a network of telescopes, with different ranges of sensitivity in the electromagnetic spectrum, within a few seconds after a neutrino identification. This scheme is particularly suited for fast transient sources [7]. Offline analyses are also possible, searching for coincidences between neutrino candidates and highly energetic events like GRBs.

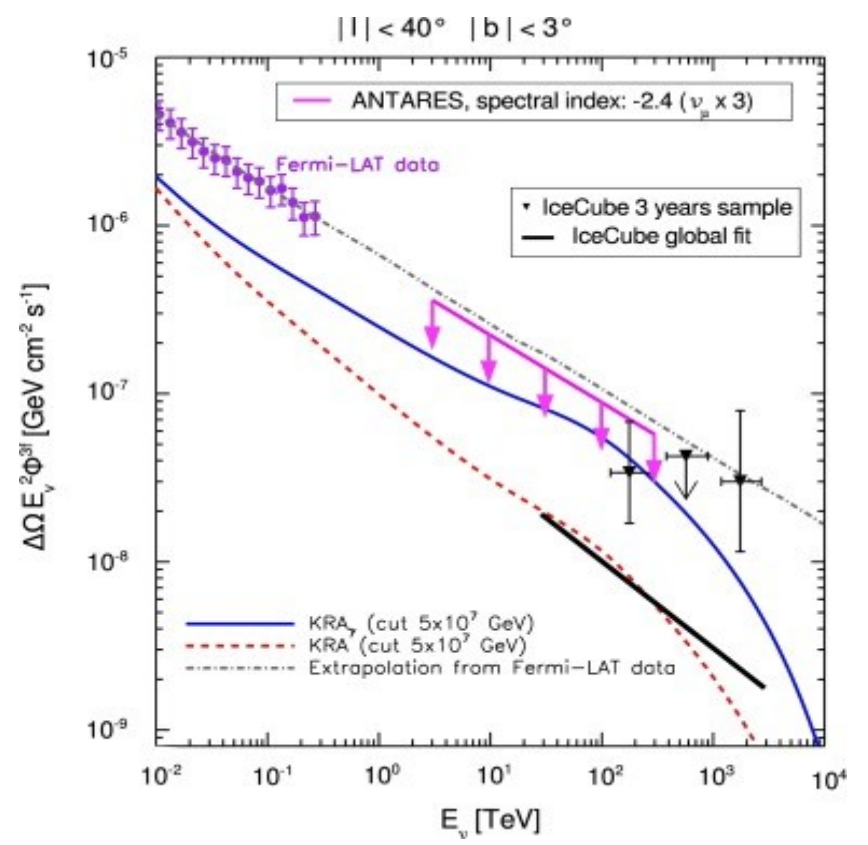

Fig. 3 - ANTARES upper limit (magenta line) on the neutrino flux integrated over the solid angle $\Delta \Omega$ $=0.145 \mathrm{sr}$ corresponding to the Galactic ridge region $|\ell|<40^{\circ},|\mathrm{b}|<3^{\circ}$. 


\section{Dark Matter}

According to a popular hypothesis, dark matter could be made of unknown, massive particles, thermal relics of the Big Bang and only weakly interacting (WIMPs). The search for these particles can be conducted via indirect detection, by means of the secondary particles produced when they decay or annihilate in pairs, subsequently producing standard model particles, including neutrinos. This kind of search with neutrino telescopes is particularly interesting because of the expected clean signal and low background [8]. Recently, an analysis has been performed looking for a neutrino flux from the Galactic Centre using track-like events collected from 2007 to 2015, for a total livetime of about 2100 days. No excess above the expected background has been measured. 90\% C.L. limits have been set for three different models of the Galactic halo, over a WIMP mass range between $50 \mathrm{GeV} / \mathrm{c}^{2}$ to $100 \mathrm{TeV} / \mathrm{c}^{2}$ and considering five decay channels. These limits are the most stringent in a certain region of the parameter space, Fig. 4.

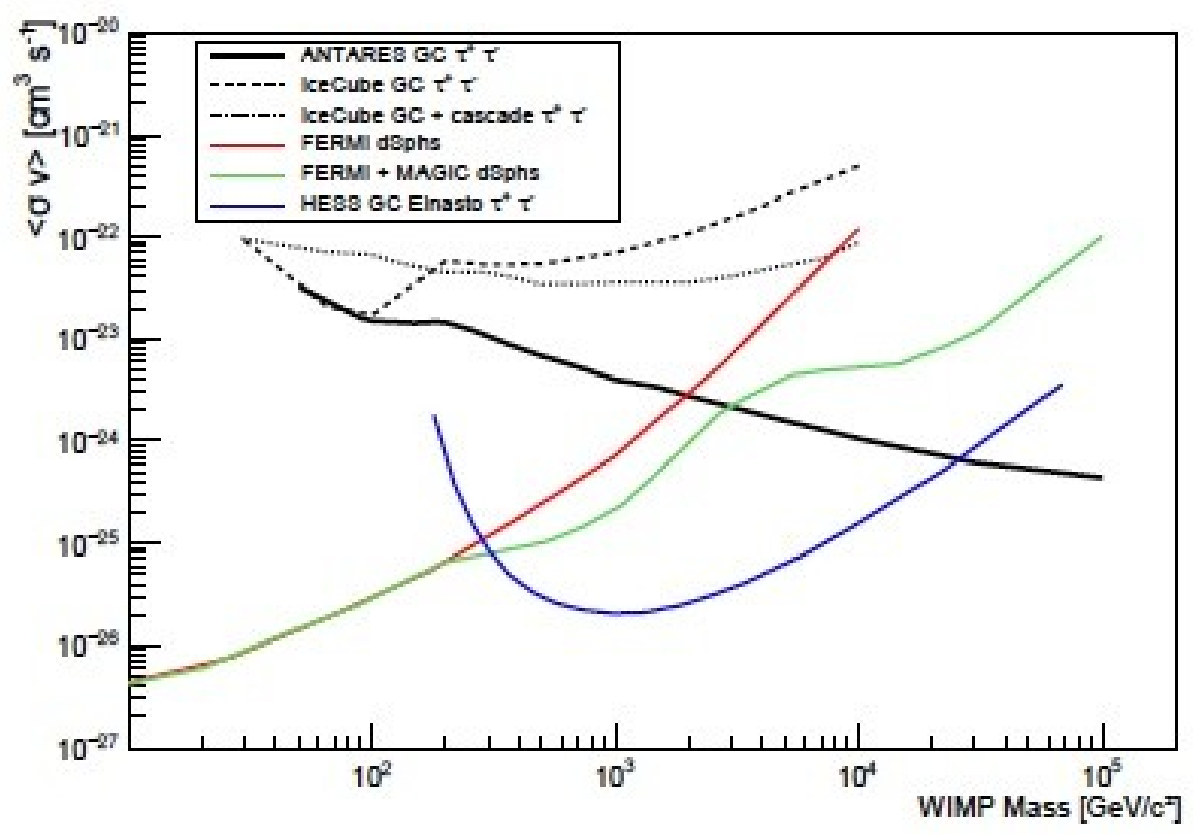

Fig. 4 - 90\% C.L. limits on the thermally averaged annihilation cross-section, as a function of the WIMP mass in comparison to the limits from other experiments.

\section{References}

[1] M. Ageron et al., NIM A 656 (2011) 11.

[2] M. G. Aartsen et al., Science 342 (2013) 1242856.

[3] S. Adrián-Martínez et al., EPJ C 73(2013) 2606; ApP 35 (2012) 634; J.A. Aguilar et al., NIM A 626-627 (2011) 128.

[4] S. Adrián-Martínez et al., ApJ Lett. 743 (2011) L14; ApJ 760 (2012) 53; ApJ Lett. 786 (2014) L5. 
[5] S. Adrián-Martínez et al., PLB 760 (2016) 143; EPJ C 74 (2014) 2701.

[6] S. Adrián-Martínez et al., PRD 93 (2016) 122010.

[7] S. Adrián-Martínez et al., JCAP 02 (2016) 062.

[8] S. Adrián-Martínez et al., PLB 759 (2016) 69; JCAP 05 (2016) 016. 